\title{
Seeing what others see while thinking what others have not
}

\section{DOI: 10.7861/clinmed.ed.21.6.1}

The characterisation of research embodied in the title is one that is especially relevant to this edition of Clin Med. The COVID-19 vaccine has been the most high-profile research output from the pandemic. The breath-taking speed of the technological advances and the clinical trials was an exemplar of how the scientific and medical communities can collaborate. The recognition of rare cases of thrombosis in association with thrombocytopenia with the Oxford-AstraZeneca vaccine has highlighted another aspect of clinical research. Those initial observations galvanised the haematology and wider medical community bringing it to the attention of the Medicines and Healthcare products Regulatory Agency. A national network to rapidly exchange information was established, out of which came high impact publications and prompted national guidance. The majority of colleagues in the expert working group were NHS consultants, not formal academics, and the whole story is an exemplar to complement the emerging Royal College of Physicians' research strategy. Tim Chevassut and colleagues review the rapid understanding of pathophysiology, and how prompt, expert guidance saved lives and consolidated the national vaccination programme., Another vaccine-related effect is reactive lymphadenopathy. Positron emission tomography - computed tomography undertaken to monitor patients with malignancy may pick up these lesions with an impact on clinical pathways. El-Sayed et al report on the patient characteristics and relationship to different vaccines; these are important data to avoid inappropriate upstaging of cancer patients in the context of the possible need to have regular boosters. ${ }^{3}$

The burgeoning and immediately translated research that has emerged as a major uptick from the pandemic has reached beyond immunology to health informatics and modelling. The group at University College London were pioneers in the use of national routine data to model the excess mortality risk in high-risk individuals for COVID-19, work that directly influenced the decision to move to lockdown in the UK. A manuscript from Banerjee and colleagues from that group addresses the issue of low public and patient involvement in creation of mortality risk tools. ${ }^{4}$ They describe an iterative online process of sustained patient and public engagement to develop a risk information tool during and beyond the pandemic. The proposed tool plainly has potential value beyond the pandemic, and signposts possible future directions for both research and clinical implementation. The tool provides a potentially responsive instrument (with regard to the disease modification provided by vaccination) that has international impact given the global extent of the pandemic.

An increasingly considered aspect of the research cycle is the altruism of patients who participate in studies, especially those in which there is no financial reward. Schwarz et al describe the recruitment process of a large multicentre international cardiology study on the efficacy of inorganic nitrate supplementation on exercise capacity of patients with stable angina. ${ }^{5}$ What they describe is that patients screened for a clinical trial undergo predictably higher levels of investigation, which may then identify sub-clinical conditions. While this may act as a motivator to attract patients, it is important to minimise overdiagnosis. Understanding the natural history of diseases and watchful waiting trials of early or ambiguous abnormalities is key, as is involvement of patients in research design. As always, the CME section is at the heart of the issue, focusing this time on respiratory medicine. Please see below for a more detailed introduction to the CME section by the guest editor, Dr Gerrard Phillips.

\section{Anton Emmanuel} Editor-in-chief

Respiratory medicine is one of the major medical specialties, accounting for approximately $30 \%$ of the acute unselected medical take. It might, therefore, surprise the reader to discover that, as a distinct specialty, respiratory medicine in the UK almost died out in the 1960s/1970s. Today, however, it is very much a vibrant specialty with a vast range of very varied conditions (too many to list here), a large research base and strong physiological links. It is also very much a craft specialty, with a significant array of procedures including more recent developments such as endobronchial ultrasound bronchoscopy, indwelling pleural catheters and local anaesthetic (medical) thoracoscopy. It accommodates clinicians with a wide range of differing interests and skills. In short, it has something for everyone.

It has been a pleasure to have been invited to curate a CME section on respiratory medicine for Clinical Medicine. I have done this in conjunction with, and with much help from, Sheila Ramjug, who is a member of the British Thoracic Society's Education and Training Committee and member of the pulmonary vascular disease specialist advisory group. I am also indebted to all the authors who have given up so much of their time to participate in this project.

It has been a formidable task to choose only six areas for which there is space here. We have deliberately omitted COVID-19, as there has been so much published on this over the last 18 months. Our selection is, rather, based on the conditions that we feel the non-respiratory clinician, particularly those doing the acute medical take and looking after internal medicine inpatients, would need to know about. Indeed, we asked that the articles be very much focused on this remit.

We very much hope that you find them informative and that you really enjoy reading them.

Gerrard Phillips

Executive medical director, Federation of Royal Colleges of Physicians, London, UK and Dorset County Hospital, Dorchester, UK

\section{References}

1 Chevassut T, Hunt B], Pavord S. VITT, COVID-19 and the Expert Haematology Panel: The story of how the UK responded to emerging cases of vaccine-induced immune thrombocytopenia and thrombosis during the vaccination programme. Clin Med 2021;21:e600-2. 
2 Toh $\mathrm{C}-\mathrm{H}$. The art and science of the thrombosis with thrombocytopenia syndrome. Clin Med 2021;21:e603.

3 El-Sayed MS, Wechie GN, Low CS et al. The incidence and duration of COVID-19 vaccine-related reactive lymphadenopathy on 18FFDG PET-CT. Clin Med 2021:21:e633-8.
4 Banerjee A, Pasea L, Manohar S et al. 'What is the risk to me from Covid?': Public involvement in providing mortality risk information for people with 'high-risk' conditions for COVID-19 (OurRisk.CoV). Clin Med 2021:21:e620-28.

5 Schwarz K, Parasuraman S, Singh S et al. The unspoken benefit of participation in a clinical trial. Clin Med 2021;21:e645-7.

\section{Members of the editorial board}

Cono Ariti

Tim Chevassut

Tahseen Chowdhury

Richard Corbett

Andrew Davies

Elaine Dennison

Arosha Dissanayake

Anton Emmanuel
Kate Evans

Anna Goodman

Tevfik Ismail

Vikas Kapil

Neelam Kumar

Yash Mahida

Martin McKee

Andrew Medford
Mehool Patel

Gerrard Phillips

Sushma Saksena

Philip Smith

Angela Star

Cameron Swift

Rhys Thomas
Trainee associate editors:

Tessa Cacciottolo

Rajan Pooni

Anenta Ramakrishnan

Trainee column editor:

Adam Truelove 